DE DE GRUYTER

OPEN

G

BULGARIAN ACADEMY OF SCIENCES

CYBERNETICS AND INFORMATION TECHNOLOGIES • Volume 15, No 5

Special Issue on Control in Transportation Systems

Sofia $\bullet 2015$

Print ISSN: 1311-9702; Online ISSN: 1314-4081

DOI: $10.1515 /$ cait-2015-0020

\title{
Traffic Flow Control in the Class of Distributed Parameters Systems
}

\author{
Emil K. Nikolov, Nina G. Nikolova \\ Technical University of Sofia, 8 Kliment Ohridski Str., Sofia 1000, Bulgaria, FA, DIA \\ Emails:nicoloff@tu-sofia.bg_ninan@tu-sofia.bg
}

\begin{abstract}
In this paper a new structure and a method are suggested for the design of a control system with a distributed parameters plant. The discussed system with distributed spatial dimensions substantially differs from the control systems with concentrated parameters plants, because in it the task of the plant spatial transition of the controlled distributed variables is structurally solved. The solution of this task is based on the usage of models and filters, included in its structure and an algorithm, through which the system has real capability for distributed plants control with the desired quality. In the paper a fractal predictive system with approximated dimensions of the spatial distribution for control of the transport traffic density in highways is designed for a particular numerical example. The system model is simulated. The characteristics of the designed system are illustrated and analyzed.
\end{abstract}

Keywords: Fractional structures with a spatial transition of the projection, predictive systems with distributed parameters, fractional approximated dimension control.

\section{Introduction}

In the references the robust Smith-predictors with fractal compensators of delay (DTC-Dead-Time Compensators) are known [1-6], in which efficiency is achieved in the control of plants with a variable delay, where $R_{\mathrm{NE}}^{S}$ is the fractional controller (Fig. 1). Possibilities for traffic flow control with robust fractional Smith-DTC predictors are shown in Fig. 2. But such a structure is not efficient because it does not account for the object management with distributed parameters. The present 
paper aims at improving the robust Smith-predictive systems by a fractal DTC (Figs 1,2) when applied in the control of plants with distributed parameters. The tasks assigned to this paper are to suggest: an improved structure of the robust fractal Smith-predictive systems; a method for analytical synthesis of the improved system; a numerical example.

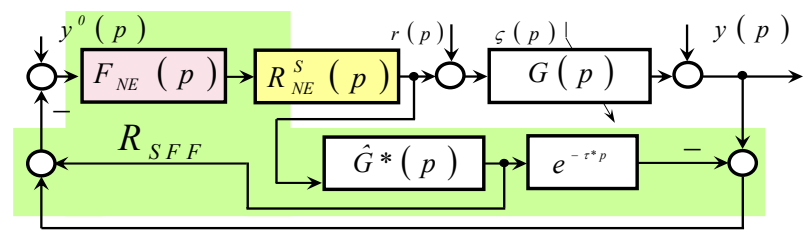

Fig. 1

\section{Design of the fractional dead-time compensators}

The rational approximation algorithm of the physically realizable DTC-filter $F_{\mathrm{NE}}$ (1) is projected analytically according to the algorithm for analytical synthesis, presented in Table 1. This algorithm realizes a polynomial recursive approximation approach for rational approximation of the fractal operator by the criterion adequacy of the frequency responses in a predefined range. A new structure is suggested (Figs 3-5) of $R_{\mathrm{NE}, \nabla}^{\mathrm{SFF}}$-system - non-integer order system for highway traffic control, substantially different from the system presented in Fig. 2. Similarly, herein the controlled distributed value is again the density $\rho_{i}(i, p)$ of the vehicle traffic in any technologically defined position on the highway. The control value is the density $\rho_{\text {entree }}(0)$ of the traffic on the highway entrance $(x=0)$. It is a uniquely determined function of the productivity and the number of the operating crossing barriers on the highway entrance, denoted by „॰”. Unlike the $R_{\mathrm{NE}}^{\mathrm{SFF}}$-system

(Fig. 2), in the control algorithm and structure of the $R_{\mathrm{NE}, \nabla}^{\mathrm{SFF}}$-system (Fig. 3), the following elements are included: $\Phi_{\mathrm{SFF}, i}^{*}$-models, $\xi$-filters $D_{i}^{\xi}, x$-filters $D_{i}{ }^{x}$, and by using them, the approximated dimension of the $R_{\mathrm{NE}, \nabla}^{\mathrm{SFF}}$-system distribution is obtained.

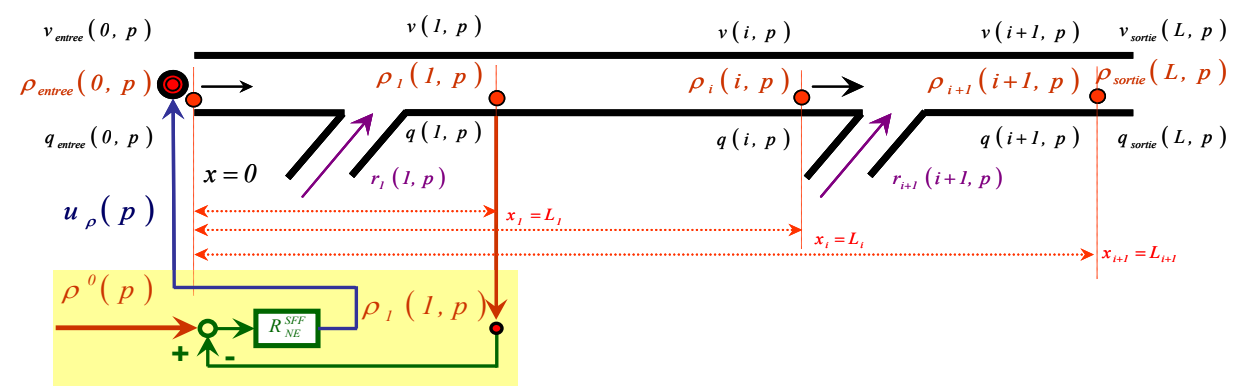

Fig. 2 
3. $\Phi_{\mathrm{SFF}, i}^{*}$-models

These $\Phi_{\mathrm{SFF}, i}^{*}$-models (2) correspond to an one-dimensional hypothetic feedback system (as the class of the presented in Fig. 2 system) for control of the nominal models $G_{i}^{*}$ of the traffic density at a particular position on the highway (selected in accordance with its particular specifics, for example, the input-output ramps) at distances $L_{i}$ from its entrance:

$$
\begin{aligned}
& F_{\mathrm{NE}} \hat{\equiv} F_{\tau} D_{\text {app }}^{\zeta}, \\
& \Phi_{\mathrm{SFF}, i}^{*}=R_{\mathrm{NE}, i}^{\mathrm{SFF}} G_{i}^{*}\left(1+R_{\mathrm{NE}, i}^{\mathrm{SFF}} G_{i}^{*}\right)^{-1}, i \geq 2 \text {, } \\
& R_{\mathrm{SFF}}=\frac{R_{\mathrm{NE}}^{S} F_{\mathrm{NE}}}{1+R_{\mathrm{NE}}^{S} \hat{G}^{*}\left(1-F_{\mathrm{NE}}^{2} e^{-p \tau^{*}}\right)}, \\
& \left(R_{\mathrm{NE}}^{S} \underset{\{\mathrm{PM} ; \mathrm{GM}=\text { const }\}}{\Leftrightarrow} \hat{G}^{*}\right) \\
& R_{\mathrm{NE}}^{\mathrm{SFF}}=\frac{(I D)_{\mathrm{app}}^{\alpha} F_{\mathrm{NE}}}{1+(I D)_{\mathrm{app}}^{\alpha} \hat{G}^{*}\left(1-F_{\mathrm{NE}}^{2} e^{-p \tau^{*}}\right)}, \\
& \left((I D)_{\mathrm{app}}^{\alpha} \underset{\{\mathrm{PM} ; \mathrm{GM}=\text { const }\}}{\Leftrightarrow} \hat{G}^{*} ; F_{\mathrm{NE}} \triangleq F_{\tau} D_{\text {app }}^{\zeta} \Leftrightarrow G^{*}\right) \text {, } \\
& 0 \mathrm{~km} / \mathrm{h} \leq v_{f} \leq 160 \mathrm{~km} / \mathrm{h} ; \\
& 0.15 \leq \rho^{0} \leq 0.4956 ; 0 \mathrm{~km} \leq L \leq 6 \mathrm{~km} \text {. }
\end{aligned}
$$

3.1. Initial conditions

It is considered that a nominal model $G^{*}(j \omega)=\hat{G}^{*}(j \omega) \exp (-j \omega \tau *)$ is known of the plant with: analytical $n$-th order model with rational and irrational components; cutoff frequency value $\omega_{C}^{*}$; value of $\arg \left(G *\left(j \omega_{\mathrm{c}}^{*}\right)\right)$ - frequency response at frequency $\omega_{\mathrm{c}}^{*}$; delay of $\tau^{*}$ value in the irrational component

\subsection{Criterion}

The criterion for approximation of $F_{\mathrm{NE}}(j \omega)=F_{\tau} D^{\zeta}(j \omega)$ is adequacy of the frequency responses of the rational system $F_{\tau} D_{\text {app }}^{\zeta}(j \omega)$ and the frequency responses of the irrational component of the nominal plant model in the predefined frequency range $\Delta \omega$,

$$
\begin{array}{cc}
\left|\exp \left(-j \omega \tau^{*}\right) F_{\tau}(j \omega) D_{\text {app }}^{\zeta}(j \omega)\right|=1, & \angle\left(\exp \left(-j \omega \tau^{*}\right) F_{\tau}(j \omega) D_{\text {app }}^{\zeta}(j \omega)\right)=0, \\
\forall \omega \in\left[\omega_{1}, \omega_{2}\right], \Delta \omega=\omega_{2}-\omega_{1}, & \forall \omega \in\left[\omega_{1}, \omega_{2}\right],\left(\Delta \omega=\omega_{2}-\omega_{1}\right) .
\end{array}
$$

\subsection{Approximation algorithm}

Step 1. The order $\zeta$ of $D_{\text {app }}^{\zeta}(j \omega)$ is determined and the time-constant $\tau_{F}$ of $F_{\tau}$ on the basis of the relations: 


$$
\begin{gathered}
\zeta \equiv\left(-\arg \left(G^{*}\left(j \omega_{\mathrm{c}}^{*}\right)\right) /(\pi / 2)\right) \rightarrow D^{\zeta}(j \omega)=\left(j \omega \omega_{u D}^{-1}\right)^{(\zeta)}, \\
\tau_{F} \equiv \tau^{*} \rightarrow F_{\tau}(j \omega)=(1+j \omega)\left(1+j \omega \tau^{*}\right)^{-1} .
\end{gathered}
$$

Step 2. The frequency $\omega_{u}$ of $D_{\text {app }}^{\zeta}(j \omega), \quad\left|D_{\text {app }}^{\zeta}\left(j \omega_{u}\right)\right|=1$ is determined according to the dependence: $1000 \omega_{\mathrm{c}}^{*} \leq \omega_{u} \leq 2000 \omega_{\mathrm{c}}^{*}$.

Step 3. After that in parallel the requirements $\zeta=\log \lambda(\log (\lambda \eta))^{-1}$; $\lambda \eta=3.58$ are applied and from them the values of $\lambda$ and $\eta$ are determined, and then by using the values of $\omega_{u}, \lambda, \eta$, the recursive parameters of the approximation $D_{\text {app }}^{\zeta}(j \omega)$ are calculated as follows:

$$
\begin{aligned}
& \omega_{A}=0.10 \omega_{u} ; \omega_{B}=10.00 \omega_{u} ; \omega_{b}=0.2 \omega_{A}=0.02 \omega_{u} ; \omega_{h}=1.2 \omega_{B}=12.00 \omega_{u} ; \\
& \omega_{1}^{\prime}=\lambda^{-0.5} \omega_{u} ; \omega_{1}=\lambda^{+0.5} \omega_{u} ; \omega_{N}=\eta^{-0.5} \omega_{h}=\eta^{-0.5} 12.00 \omega_{u} \text {, } \\
& F_{\mathrm{NE}}(j \omega) \hat{\equiv} F_{\tau} D_{\mathrm{app}}^{\zeta} \equiv \frac{1+j \omega}{1+j \omega \tau}\left(\frac{\omega_{u}}{\omega_{h}}\right)^{\zeta} \prod_{i=1}^{N}\left(1+j \frac{\omega}{\omega_{i}^{\prime}}\right)\left(1+j \frac{\omega}{\omega_{i}}\right)^{-1} .
\end{aligned}
$$

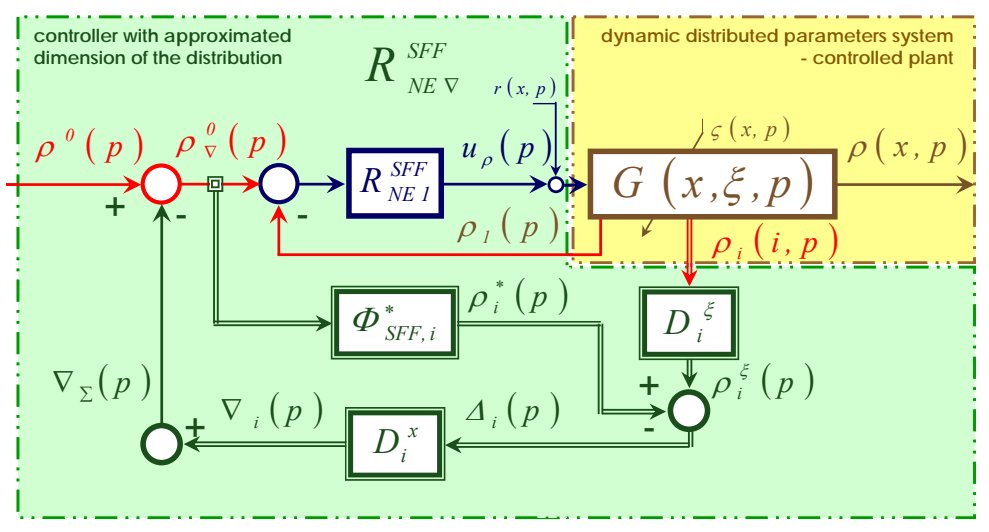

The complex non-integer-order controllers $R_{\mathrm{NE}, i}^{\mathrm{SFF}}$ (3), (4), (5) in the structure of $R_{\mathrm{NE}, \nabla}^{\mathrm{SFF}}$ (Figs 3-5) are with zero-dimension of the distribution and in the class of Smith-predictors of non-integer order $\alpha$ with fractal robust DTC-filters $F_{\mathrm{NE}, i}$ are designed.

The filters $F_{\mathrm{N} E, i}$ approximate rationally, in a restricted frequency range, the operators for a fractional-order $\zeta$ see differentiation (6), (7) as fulfillment of the requirement (8) for time delay compensation.

In $R_{\mathrm{NE}, i}^{\mathrm{SFF}}$ (see (9)) the design of the corresponding nominal models $G_{i}{ }^{*}(i, p)$ (see(10)) at particular plant positions $i$ for the corresponding distances, the criteria vertical profile and adequacy of the frequency responses in a predefined range are applied [5]. 
The input reference value $\rho^{0}$ is the same as the main $R_{\mathrm{NE}, 1}^{\mathrm{SFF}}, i=1$ controller, like into the rest complex $R_{\mathrm{NE}, i}^{\mathrm{SFF}}, i \geq 2$ controllers in the nominal models $\Phi_{\mathrm{SFF}, i}^{*}$ of the control system. The main controller $R_{\mathrm{NE}, 1}^{\mathrm{SFF}}$, (the closest to the highway entrance) generates the control input $u_{\rho}$ (a concentrated value) to the system at the entrance of the highway:

$$
\begin{aligned}
D^{\zeta}(j \omega) & =\left(j \omega \omega_{u D}^{-1}\right)^{(\zeta)}, \omega_{u D}=T_{d}^{-\zeta} \\
D^{\zeta}(j \omega) & \cong D_{\mathrm{app}}^{\zeta}(j \omega), \quad \omega \in\left[\omega_{A}, \omega_{B}\right], \\
& e^{-p \tau^{*}} F_{\mathrm{NE}}(p) \equiv 1 .
\end{aligned}
$$

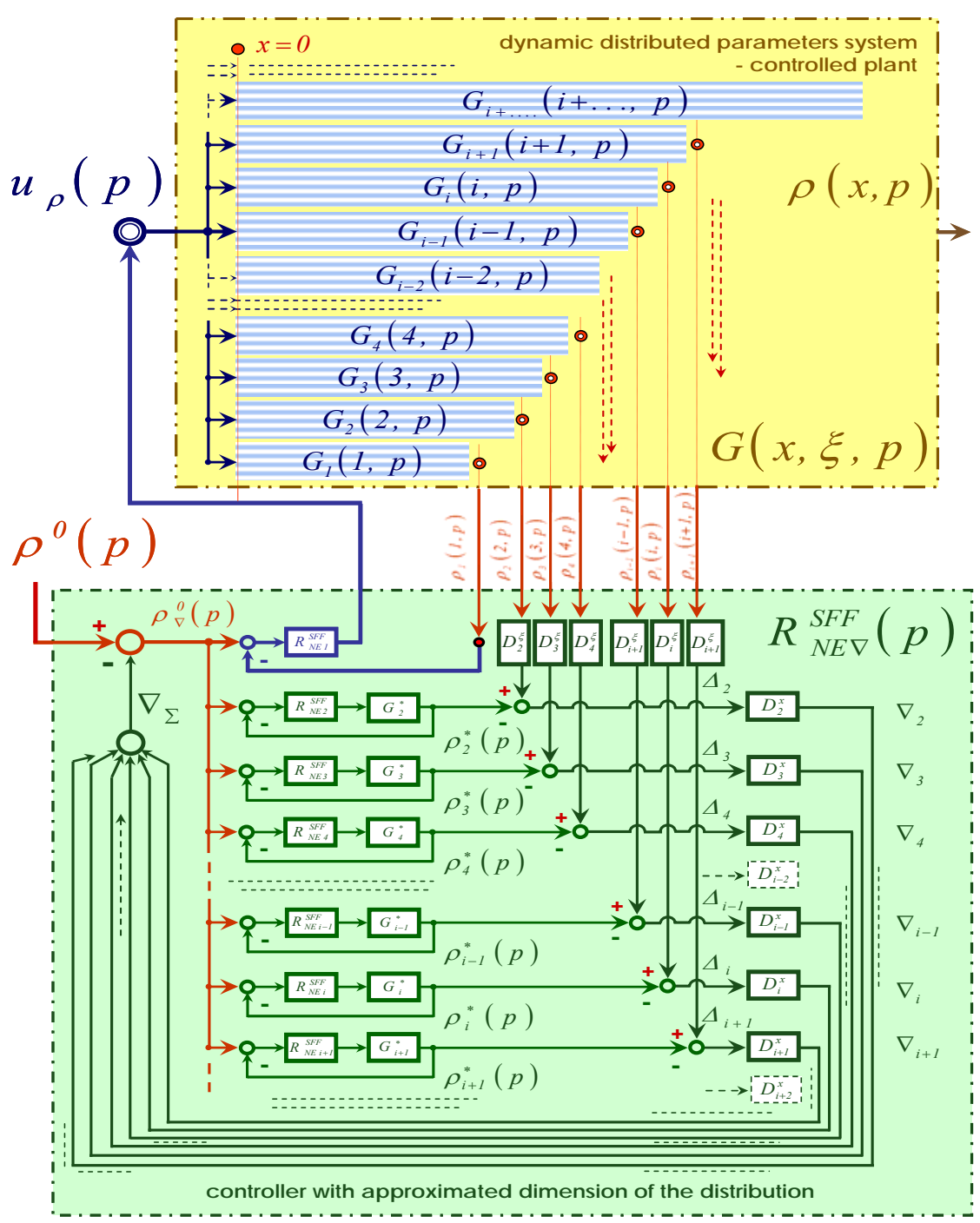

Fig. 4 


\section{4. $\xi$-filers $D_{i}^{\xi}$}

The really measured traffic densities $\rho_{i}(i, p)$ at the corresponding positions $i$ on the highway (Figs 3-5) are transformed in scalar values $\rho_{i}^{\xi}(p)$ by using the $\xi$-filters $D_{i}^{\xi}$ (see (11)).

By their characteristics, the $\xi$-filters $D_{i}^{\xi}$ operate as spatial transition of the projection. $\xi$-filters are a core of the control system, which is independent on the dimension and the input spatial variable; it depends only on the output spatial variable $\xi$ of the distributed system. They are intended for achievement of approximated spatial dimension of the distribution of the controller $R_{\mathrm{NE}, \nabla}^{\mathrm{SFF}}(p)$ to the controlled plant $G(x, \xi, p)$.

On this basis, the $\xi$-filters $D_{i}^{\xi}$ design (see Fig. 5) is made according to the values of the time-constants $T_{q, i}$ and $T_{r, i}$ (see (12)) and is realized in accordance with (11).

\section{5. $x$-filers $D_{i}{ }^{x}$}

The differences $\Delta_{i}$ (13) between the "concentrated" output values $\rho_{i}^{*}$ of the models $\Phi_{\mathrm{SFF}, i}^{*}$ (Fig. 3) and the transformed into scalars values $\rho_{i}^{\xi}(p)$ of the really measured, at the corresponding positions $i$ on the highway, traffic densities $\rho_{i}(i, p)$ form the input variables of $x$-filters $D_{i}{ }^{x}$ (14) for the approximated spatial dimension of the distribution of controller $R_{\mathrm{NE}, \nabla}^{\mathrm{SFF}}$ in the control system to the plant under control. By its characteristics, $x$-filters $D_{i}^{x}$ operate as "spatial transition of the projection".

They are the core of the control system, which is independent of the input spatial variables, it depends solely and only on the spatial variable $x$.

On this basis, $x$-filters $D_{i}^{x}$ (Fig. 5) design is made in accordance with the values of the time-constants $T_{k, i}$ and $T_{l, i}(15)$ :

$$
\begin{gathered}
R_{\mathrm{NE}, i}^{\mathrm{SFF}}(p)=\frac{(I D)_{\mathrm{app}, i}^{\alpha} F_{\mathrm{NE}, i}(p)}{1+(I D)_{\mathrm{app}, i}^{\alpha} \hat{G}_{i}^{*}(p)\left(1-F_{\mathrm{NE}, i}^{2}(p) e^{\left.-p \tau^{*}\right)}\right.}, \\
\left((I D)_{\mathrm{app}, i}^{\alpha} \underset{\left\{\mathrm{PM} ; \mathrm{GM}=\mathrm{const}_{i} \hat{G}_{i}^{*} ; F_{\mathrm{NE}, i} \hat{\equiv} F_{\tau} D_{\mathrm{app}, i}^{\zeta} \Leftrightarrow G_{i}^{*}\right),}{\left(T\left(i, \xi, v_{f}, \rho_{0}\right) p+1\right)} e^{-\tau\left(i, \xi, v_{f}, \rho_{0}\right) p},\right.
\end{gathered}
$$




$$
\begin{gathered}
D_{i}^{\xi}(p)=\rho_{i}(i, p) / \rho_{i}^{\xi}(p)=\left(T_{q} p+1\right)\left(T_{r} p+1\right)^{-1}, \quad i \geq 2, \\
T_{q, i} \geq \tau_{i}^{*}, T_{r, i}=\tau_{i}^{*} \cong T_{q, i}, \quad i \geq 2,
\end{gathered}
$$

$$
\Delta_{i}(p)=\rho_{i}^{\xi}(p)-\rho_{i}^{*}(p), i \geq 2,
$$

$$
D_{i}^{x}(p)=\nabla_{i}(p) / \Delta_{i}(p)=\left(T_{k} p+1\right)\left(T_{l} p+1\right)^{-1}, i \geq 2 \text {, }
$$

$$
T_{k, i} \geq 10 L \mathrm{~s}, \quad T_{l, i}=\tau_{i}^{*}<<T_{k, i}, \quad i \geq 2 \text {. }
$$
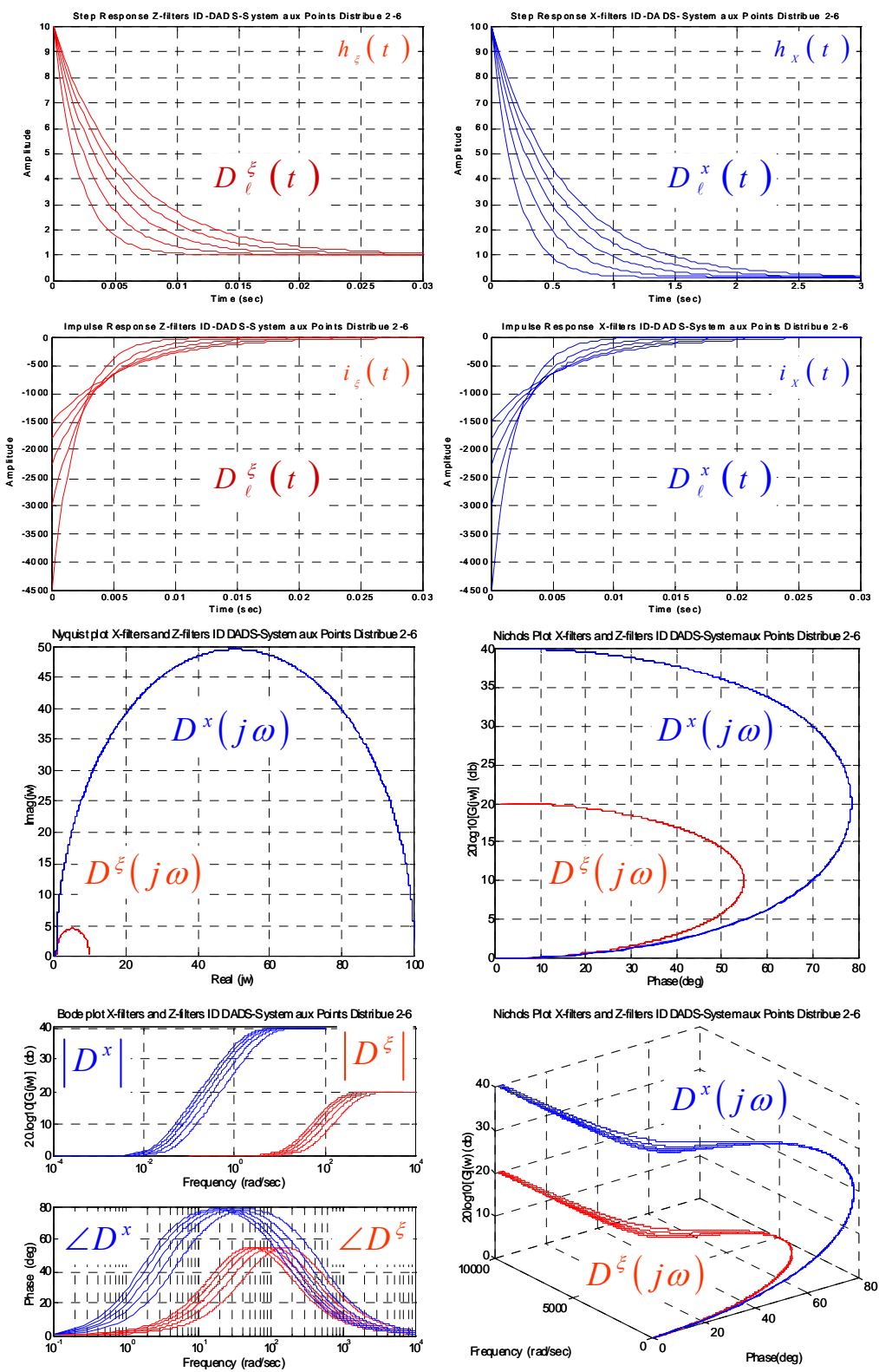

Fig. 5 


\section{A controller with approximated spatial dimenstion}

\section{of the distribution}

By using the $D_{i}^{x}$ outputs $\nabla_{i}, i \geq 2$, additively correcting the reference value $\rho^{0}$ by subtraction of $\nabla_{\Sigma}$ (16), in the $R_{\mathrm{NE}, \nabla}^{\mathrm{SFF}}$-system (Figs 3-5) approximated dimension of the distribution of the controlling component $R_{\mathrm{NE}, \nabla}^{\mathrm{SFF}}$ (controller with approximated spatial dimension of the distribution) and of the controlled dynamic system (the traffic) is achieved. The controller $R_{\mathrm{NE}, \nabla}^{\mathrm{SFF}}$ generates a control input $u_{\rho}$, which is concentrated by its nature, and is analytically described by (17)-(22), but it detects and efficiently corrects the changes and disturbances in the system with spatial distribution of the parameters. This is possible by using the $\Phi_{\mathrm{SF}, i}^{*}$-models, $\xi$-filters $D_{i}^{\xi}$ and $x$-filters $D_{i}^{x}$ in the structure of $R_{\mathrm{NE}, \nabla}^{\mathrm{SFF}}$.

$$
\begin{aligned}
& \nabla_{\Sigma}(p)=\sum_{i} \nabla_{i}(p), i \geq 2, \\
& u_{\rho}=\rho_{\nabla}^{0} R_{\mathrm{NE}, 1}^{\mathrm{SFF}} \text {, } \\
& \rho_{\nabla}^{0}=\rho^{0}-\sum_{\ell=2}^{i} \nabla_{\ell}, \\
& \nabla_{\ell}=\left(D_{l}^{\xi} \rho_{l}(l, p)-\rho_{\nabla}^{0} R_{\mathrm{NE}, l}^{\mathrm{SFF}} G_{l}^{*}\left(1+R_{\mathrm{NE}, l}^{\mathrm{SFF}} G_{l}^{*}\right)^{-1}\right) D_{l}^{x}, \\
& \rho_{\nabla}^{0}=\left(\rho^{0}-\sum_{l=2}^{i} D_{l}^{\xi} D_{l}^{x} \rho_{l}(l, p)\right), \\
& \left(1-\sum_{l=2}^{i} D_{l}^{x} R_{\mathrm{NE}, l}^{\mathrm{SFF}} G_{l}^{*}\left(1+R_{\mathrm{NE}, i}^{\mathrm{SFF}} G_{l}^{*}\right)^{-1}\right)^{-1}, \\
& u_{\rho}=\left(\rho^{0}-\sum_{l=2}^{i} D_{l}^{\xi} D_{l}^{x} \rho_{l}(l, p)\right), \\
& R_{\mathrm{NE}, 1}^{\mathrm{SFF}}\left(1-\sum_{l=2}^{i} D_{l}^{x} R_{\mathrm{NE}, l}^{\mathrm{SFF}} G_{l}^{*}\left(1+R_{\mathrm{NE}, i}^{\mathrm{SFF}} G_{l}^{*}\right)^{-1}\right)^{-1}, \\
& u_{\rho}=\left(\rho^{0}-\sum_{l=2}^{i} D_{l}^{\xi} D_{l}^{x} \rho_{l}(l, p)\right)(I D)_{\mathrm{app}, 1}^{\alpha} F_{\mathrm{NE}, 1}, \\
& \left(\left(1-\sum_{l=2}^{i} D_{l}^{x} R_{\mathrm{NE}, l}^{\mathrm{SF}} G_{l}^{*}\left(1+R_{\mathrm{NE}, i}^{\mathrm{SFF}} G_{l}^{*}\right)^{-1}\right),\right. \\
& \left.\left(1+(I D)_{\mathrm{app}, 1}^{\alpha} \hat{G}_{1}^{*}\left(1-F_{\mathrm{NE}, 1}^{2} e^{-p \tau^{*}}\right)\right)\right)^{-1} .
\end{aligned}
$$




\section{Numerical example, analysis and quantitive estimation of the quality}

In the range of parametric fluctuations (5), the following components are designed: - $R_{\mathrm{NE}, i}^{\mathrm{SFF}}$ Smith-predictors of non-integer order $\alpha$ with fractal robust DTC-filters $F_{\mathrm{NE} i}$ of fractal order $\zeta$ according to (9); $\bullet \Phi_{\mathrm{SFF}, i}^{*}$-models according to (2); $\xi$-filters $D_{i}^{\xi}$ in accordance with (11), (12); $x$-filters $D_{i}^{x}$ according to (14), (15), in a general structure (Figs 3-5) of the predictive $R_{\mathrm{NE}, \nabla}^{\mathrm{SFF}}$-system with approximated dimension of the distribution for traffic control.

The designed $R_{\mathrm{NE}, \nabla}^{\mathrm{SFF}}$-system is modeled. For a particular numerical example with mode dependent change of the reference input value $\rho^{0}$ to the system and disturbance inputs $r_{i}$, fed simultaneously to five neighbour positions from $i$ up to $i+4$ at the highway, as results from the simulation of the designed $R_{\mathrm{NE}, \nabla}^{\mathrm{SFF}}$-system model the following elements are presented in Fig. 6: the corrections $\nabla_{i}$ up to $\nabla_{i+4}$, generated by $R_{\mathrm{NE}, \nabla}^{\mathrm{SFF}}$-system additively with an opposite sign to the input reference value $\rho^{0}$ from $R_{\mathrm{NE}, \nabla}^{\mathrm{SFF}}$ to form the corresponding operating reference $\rho_{\nabla}^{0}$ to the compound controllers $R_{\mathrm{NE}, \nabla}^{\mathrm{SFF}}$; the resulting reference values $\rho_{i}^{0}$ up to $\rho_{i+4}^{0}$ to the main and to the compound controllers in $R_{\mathrm{NE}, \nabla}^{\mathrm{SFF}}$-system, corresponding to the corrections from $\nabla_{i}$ up to $\nabla_{i+4}$; the summarized additive $\nabla_{\Sigma}=\nabla_{i}+\nabla_{i+1}+\nabla_{i+2}+\nabla_{i+3}+\nabla_{i+4}$ correction (16), by which the $R_{\mathrm{NE}, \nabla}^{\mathrm{SFF}}$-system with approximated dimension of the distribution compensates the disturbances $r_{i}$ by using the corresponding corrections in the input reference value $\rho^{0}$ to $\rho_{\nabla}^{0}$; the resulting operative reference value $\rho_{\nabla}^{0}$ as a result of the summarized additive correction $\nabla_{\Sigma}$ to the compound controllers of $R_{\mathrm{NE}, \nabla}^{\mathrm{SF}}$.

In general, the processes, presented in Fig. 4, illustrate the operational "technology" of the $R_{\mathrm{NE}, \nabla}^{\mathrm{SFF}}$-system with approximated dimension of the spatial distribution. The system (Figs 3-5) realizes partly (and in this sense approximately) an equivalent spatial dimension of the $R_{\mathrm{NE}, \nabla}^{\mathrm{SFF}}$-controller and of the controlled plant. Fully equivalent dimension of the controlled and controlling components of the system is unachievable. 


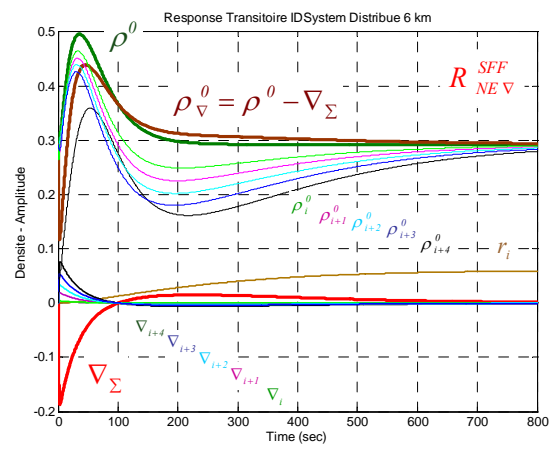

Fig. 6

The main responses of the $R_{\mathrm{NE}, \nabla}^{\mathrm{SFF}}$-system with approximated dimension of the spatial distribution (Figs 3-5), through results from the model simulation, are presented in Figs as follows:

- in Fig. 7 - the step response $\rho_{i}(t, \varsigma)$ of the traffic density in the closed-loop system $\Phi_{\mathrm{NE}, \nabla}^{\mathrm{SFF}}(t, \varsigma)$, including the nominal $\rho_{i}^{*}(t)$ of the step input reference $\rho^{0}=0.45$, with load $r_{i}$ with a positive and negative sign, applied separately to the positions in the highway at distances $2 \mathrm{nd}, 3 \mathrm{rd}, 4 \mathrm{th}, 5$ th and 6 th kilometer from the entrance;

- in Fig. 8 - the step response $\rho_{i}(t, \varsigma)$ of the traffic density in the closedloop system $\Phi_{\mathrm{NE}, \nabla}^{\mathrm{SFF}}(t, \varsigma)$, including the nominal $\rho_{i}^{*}(t)$, with a mode dependent change of the reference $\rho^{0}$, simulating the peak traffic modes, with load $r_{i}$, applied separately to the positions in the highway at distances 2nd, 3rd, 4th, 5th and 6th kilometer from the entrance;

- in Figs 9,10 - the step response $\rho_{i}(t, \varsigma)$ of the traffic density in the closed-loop system $\Phi_{\mathrm{NE}, \nabla}^{\mathrm{SFF}}(t, \varsigma)$, including the nominal $\rho_{i}^{*}(t)$, of the step input reference $\rho^{0}=0.45$, with variable load $r_{i}$, applied simultaneously to the positions in the highway at distances $2 \mathrm{nd}, 3 \mathrm{rd}, 4$ th, 5 th and 6 th kilometer from the entrance;

- in Fig. 11 - the step response $\rho_{i}(t, \varsigma)$ of the traffic density in the closedloop system $\Phi \underset{\mathrm{NE}, \nabla}{\mathrm{SFF}}(t, \varsigma)$, including the nominal $\rho_{i}^{*}(t)$, with a mode dependent change of the reference input value $\rho^{0}$, simulating peak traffic modes, with variable load $r_{i}$, applied simultaneously to the positions in the highway at distances 2nd, 3rd, 4th, 5th and 6th kilometer from the entrance;

- in Fig. 12 - the step response $\rho_{i}(t, \varsigma)$ of the traffic density in the closedloop system $\Phi_{\mathrm{NE}, \nabla}^{\mathrm{SFF}}(t, \varsigma)$, including the nominal $\rho_{i}^{*}(t)$, with a mode dependent 
change of the reference input value (input signal) $\rho^{0}$ in the described conditions for the possible fluctuation in the initial conditions.

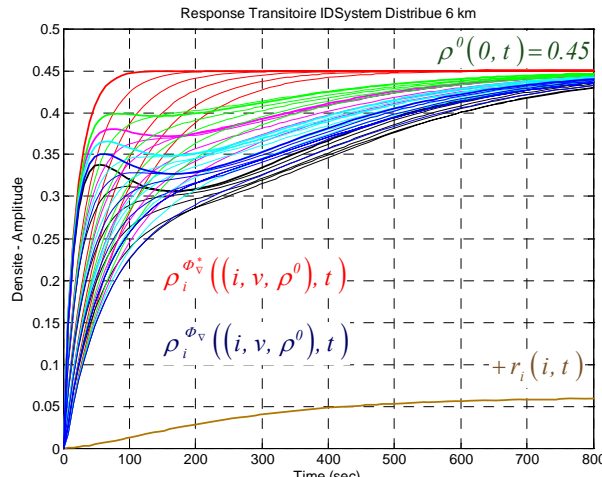

Fig. 7

constant, mode dependent input reference nominal system without load with load on the $2 \mathrm{~d} \mathrm{~km}$; with load on the $3 \mathrm{~d} \mathrm{~km}$; with load on the 6 th $\mathrm{km}$;

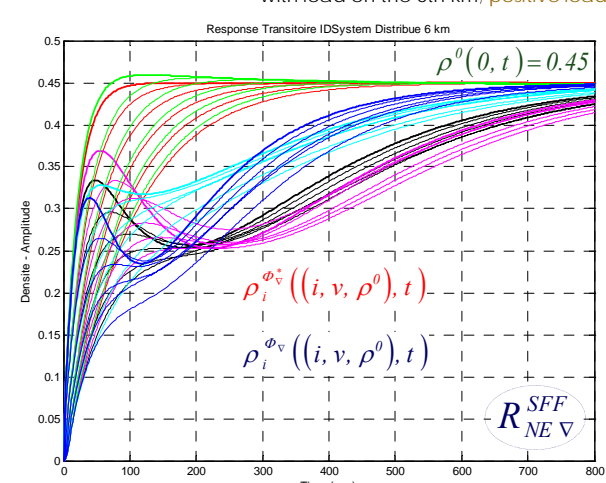

Fig. 9 constant, mode dependent input reference nominal system without load
with load on the $2 \mathrm{~d} \mathrm{~km}$; with load on the $3 \mathrm{~d} \mathrm{~km}$; with load on the $2 \mathrm{~d} \mathrm{~km}$; with load on the $3 \mathrm{~d} \mathrm{~km}$; with load on the 6 th $\mathrm{km}$; position dependent load Response Transitoire IDSystem Distribue $6 \mathrm{~km}$

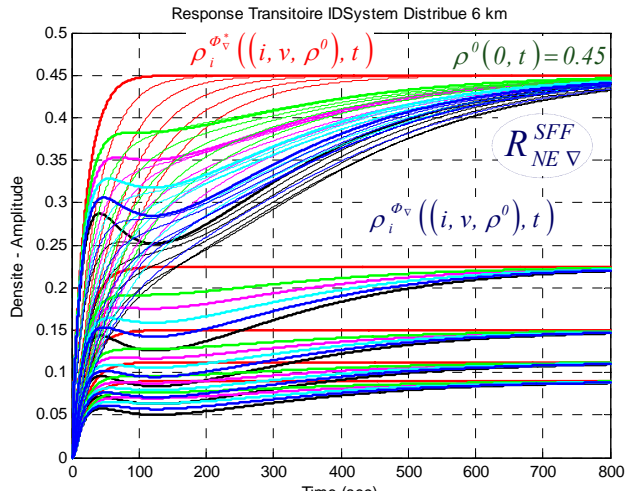

Fig. 11

constant mode dependent input reference with load on the 2 nominal system without load with load on the 5 th $\mathrm{km}$ : with load on the 6th $\mathrm{km}$; positive load

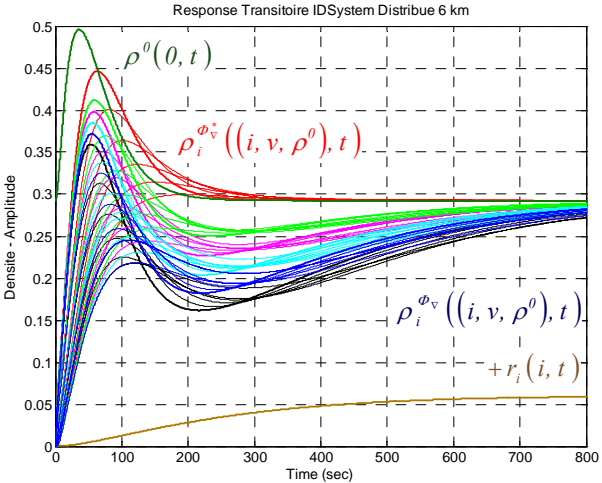

variable, mode dependent input reference nominal system without load

Fig. 8

with load on the $2 \mathrm{~d} \mathrm{~km}$; with load on the $3 \mathrm{~d} \mathrm{~km}$;

with load on the 4 th $\mathrm{km}$; with load on the 5 th $\mathrm{km}$; with load on the 6th $\mathrm{km}$; positive load

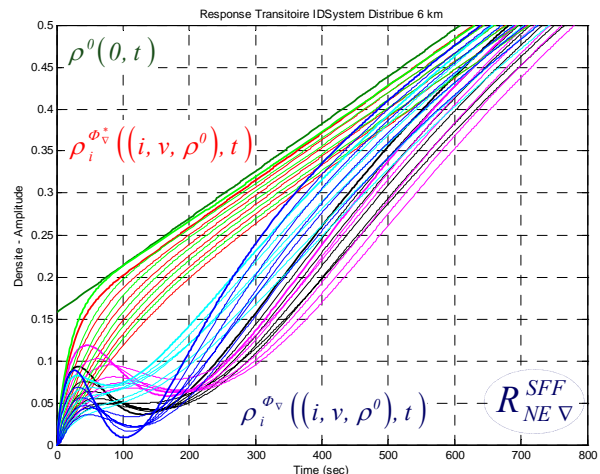

variable, mode dependent input reference

with load on the $2 \mathrm{~d} \mathrm{~km}$; with load on the $3 \mathrm{~d} \mathrm{~km}$;

Fig. 10

with load on the $4 \mathrm{~km}$; w with load on the 5 th $\mathrm{km}$;

with load on the 6 th $\mathrm{km}$; position dependent load

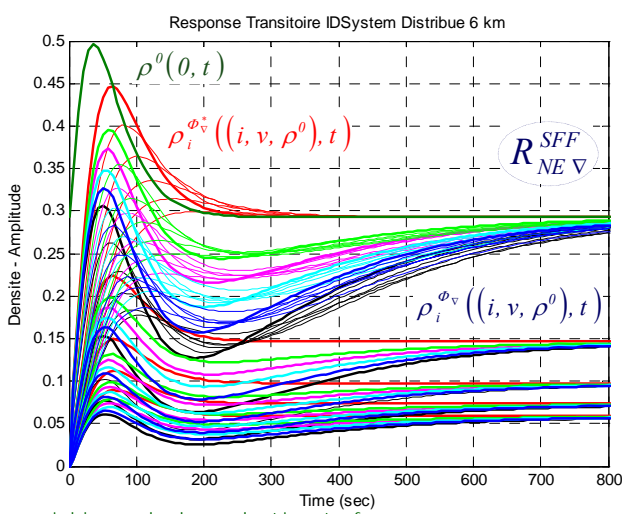

variable, mode dependent input reference nominal system without load

with load on the $2 \mathrm{~d} \mathrm{~km}$; with load on the $3 \mathrm{~d} \mathrm{~km}$;

Fig. 12

with load on the $2 \mathrm{~d} \mathrm{~km}$; with load on the $3 \mathrm{~d} \mathrm{~km}$;

with load on the 6 th $\mathrm{km}$ : positive loa 


\section{Conclusion}

The claims of this first part of the research are expressed by the suggested new structure (Figs 3-5) of the non-integer order $R_{\mathrm{NE}, \nabla}^{\mathrm{SFF}}$ - system for control of the plants with distributed parameters (highway traffic) and analytical synthesis of the improved system. Examination of the basic characteristics, estimation of the applicability and analysis of the efficiency of non-integer order $R_{\mathrm{NE}, \nabla}^{\mathrm{SFF}}$-systems are an object of the second part of the present elaboration.

Acknowledgements: This paper is partially supported by FP7 Project 316087 ACOMIN: Advance Computing and Innovation.

\section{References}

1. B o u c h e r, P., D. D i m u r. La commande prédictive. Hermès-Lavoisier, 2006. 230 p.

2. Butkovskiy, A. G. Distributed Control Systems. American Elsevier Publishing Company, 1969. $310 \mathrm{p}$

3. C a m a c ho, E. F., C. B or d o n s. Model Predictive Control. Second Ed. Springer-Verlag, 2004. $326 \mathrm{p}$.

4. M a c i e j o w s k i, J. M. Predictive Control with Constraints. Prentice Hall, London, 2002. 514 p.

5. Nikolov, E. Robust Fractional Control (Approaches Predictive and Algebraic, Distributed Control Systems), Sofia, Publishing House of Technical University of Sofia, 2010, ISBN 978-954-438-851-5. $375 \mathrm{p}$.

6. P a l mor, Z. J. The Control Handbook, Time Delay Compensation: Smith Predictor and its Modifications. CRC Press and IEEE Press, 1996. 320 p. 